\title{
Influence of Cystic Fibrosis Plasma on Lymphocyte Responses to Pseudomonas Aeruginosa In Vitro
}

\author{
RICARDO U. SORENSEN, ${ }^{(4, i)}$ PATRICIA A. CHASE, ROBERT C. STERN, AND STEPHEN H. POLMAR \\ Departments of Pediatrics and Pathology, Case Western Reserve University School of Medicine, and Rainbow Babies \\ and Children's Hospital. Cleveland, Ohio (USA)
}

\begin{abstract}
Summary
Peripheral blood lymphocytes from cystic fibrosis (CF) patients with advanced diseases do not proliferate following exposure to Pseudomonas aeruginosa (PA) antigens in in vitro. In this study, we sought to determine if $C F$ lymphocyte unresponsiveness to $P A$ is due to inhibitory factors present in CF plasma. Nineteen lowresponder CF (LRCF) patients increased their mean lymphocyte proliferative response $\left({ }^{3} \mathrm{H} \mid\right.$ thymidine incorporation) from $703 \pm$ 133 to $3178 \pm 811$ net $\mathrm{cpm}$ when incubated in normal plasma. These increases do not reach the level of response seen in normal individuals $(8510 \pm 1323$ net $\mathrm{cpm})$. Ten of 19 patients did not increase their responses over $\mathbf{2 0 0 0}$ net cpm. Plasma from LRCF patients does not inhibit responses of normal or homologous $\mathrm{CF}$ lymphocytes. Responses of normal individuals in autologous plasma were $7807 \pm 1164$ net $\mathrm{cpm}$. The same lymphocytes incubated in 16 plasmas from LRCF patients gave responses of 7146 \pm 1317 net cpm. Preincubation of the PA antigen in LRCF plasma increases rather than inhibits normal lymphocyte responses. LRCF plasma absorbed with PA no longer supports normal lymphocyte responses to PA. LRCF and normal plasma mixtures increase responses of normal lymphocytes to PA over responses in autologous plasma. Extensive preincubation and washing of LRCF lymphocytes to eliminate blocking immune complexes failed to restore the ability to respond to PA. These data suggest that the unresponsiveness to $\mathrm{PA}$ of lymphocytes from $\mathrm{CF}$ patients with advanced disease is due to alterations occurring at a cellular level in vivo. This lymphocyte dysfunction cannot be reversed by normal plasma in vitro, nor can it be induced in normal lymphocytes by the use of CF plasma.
\end{abstract}

\section{Speculation}

Lymphocytes from cystic fibrosis (CF) patients with advanced disease fail to proliferate in response to Pseudomonas aeruginosa antigens. This abnormality cannot be reversed by the use of normal plasma or by preincubation and washing of CF lymphocytes in vitro. Heat-inactivated $C F$ plasma does not inhibit proliferation of normal lymphocytes in response to Pseudomonas. These results indicate that changes in lymphocyte reactivity occur in vivo and are unresponsive to plasma substitution in vitro. Further investigation of quantitative and functional aspects of lymphocyte and macrophage subpopulations may elucidate the nature and significance of the unresponsiveness of CF lymphocytes to Pseudomonas aeruginosa.

Patients with cystic fibrosis (CF) are unusually susceptible to chronic pulmonary infection with Pseudomonas aeruginosa (PA), a ubiquitous organism which rarely infects healthy individuals (5. 14). The cause of persistent PA infection in the bronchopulmonary tract of CF patients remains unexplained. Defective mucociliary transport has been detected in some CF patients, but this does not explain the predilection for PA infection over other common pulmonary pathogens $(32,34)$. Pulmonary alveolar macrophages and neutrophils play an important role in clearing bacteria from the lung. The phagocytic function of these cells is nonspecific, but can be enhanced by antibodies and by lymphokines produced by lymphocytes specifically stimulated by antigens $(8,11,12,15,28)$. Therefore, if a specific immune defect is responsible for PA infections in CF, it is likely to occur at the lymphocyte level. We have reported a CF-specific lymphocyte unresponsiveness to PA and other gram-negative bacteria in vitro $(22,23)$. In some patients, this defect was less pronounced when the lymphocytes were incubated in normal plasma. Conversely, in some instances, plasma from an unresponsive $C F$ sibling reduced the lymphocyte response to PA of the responding CF sibling (24). These findings suggested the presence of heat-stable lymphocyte-inhibiting factors in the plasma of CF patients. A heat-stable serum factor has been shown to cause specific inhibition of PA phagocytosis by rabbit and human alveolar macrophages (26). In this study, we explored the possibility that plasma factors similar or identical to those reported to inhibit macrophages could also cause the lymphocyte unresponsiveness to $\mathrm{PA}$ in CF patients.

\section{MATERIALS AND METHODS}

\section{IN VITRO LYMPHOCYTE PROLIFERATION ASSAY}

Mononuclear cells consisting of approximately $90 \%$ lymphocytes were isolated from heparinized peripheral blood by FicollHypaque centrifugation (27). Lymphocyte proliferation studies were performed as perviously described $(23,24)$. Mononuclear cells at a concentration of $10^{5} / 0.1 \mathrm{ml}$ in Roswell Park Memorial Institute (RPMI) 1640 culture medium supplemented with $20 \%$ heat-inactivated plasma. 4-(2-hydroxyethyl)-piperazineethanesulfonic acid buffer, and antibiotics were incubated in flat-bottom microculture plates. Ten $\mu$ l of antigen (bacterial suspension) were added to each culture. Cultures were incubated at $37^{\circ} \mathrm{C}$ for 5 days and then pulsed with $0.5 \mu \mathrm{Ci}$ of $\left[{ }^{3} \mathrm{H}\right]$ thymidine (specific activity, $24 \mathrm{Ci} / \mathrm{mmole}$ ) $5 \mathrm{hr}$ before the end of the incubation period. The cells were harvested with a lymphocyte microharvester (Otto Hiller Co., Madison, WI). Incorporation of tritiated thymidine was measured with a Searle Isocap 300 liquid scintillation counter. Proliferative responses were expressed as cpm.

\section{BACTERIAL ANTIGEN PREPARATION}

Bacterial antigens were prepared by using a modification of a previously described method (22). Well-identified and pure bacterial strains were grown under optimal conditions in trypticase soy broth without dextrose and then killed by the addition of gentamicin. The antibiotic-killed bacteria were washed by repeated centrifugation with sterile isotonic saline, diluted to a $10 \%$ $(\mathrm{v} / \mathrm{v})$ stock suspension in saline, and stored at $4^{\circ} \mathrm{C}$. Before use, the supernatant was discarded, and the bacterial pellet was resus- 
pended to the original concentration in fresh saline; $1,0.1,0.01$, and $0.001 \%$ dilutions were used in lymphocyte culture assays.

Clinical isolates of $P$. aeruginosa (one classic strain and one mucoid strain), and Cowan 1 strain of Staphllococcus aureus (American Type Culture Collection), were prepared as antigens. Fresh antigens were prepard every 2 months from the original strains and tested before use with normal lymphocytes of known reactivity. All bacterial preparations were shown to be sterile before use.

\section{PATIENTS AND NORMAL ('ONTROLS}

Healthy young adults served as controls. CF was diagnosed by a positive sweat test and either typical pulmonary or gastrointestinal manifestations or a family history of CF. We have previously shown that there is a significant correlation between unresponsiveness to PA and low case history scores in CF patients (22-24). To analyze the effect of plasma factors, both normal controls and $C F$ patients were grouped into three categories according to their responses to PA in autologous plasma: low responders $<2000$; medium responders, 2000 to 8000 ; and high responders $>8000$ net cpm. In experiments involving CF lymphocytes, at least one normal control was always included. These studies were approved by the Clinical Research Review Committee of University Hospitals of Cleveland, and written informed consent was obtained.

\section{PLASMA PREPARATION}

Plasma was obtained from heparinized peripheral blood by 20 min centrifugation for $20 \mathrm{~min}$ at $800 \times \mathrm{g}$. Plasma samples were then heat inactivated for $30 \mathrm{~min}$ at $56^{\circ} \mathrm{C}$ and spun again for 30 $\min$ at $1600 \times g$. In most instances, the plasma substitution experiments were performed on the same day, and the remaining plasma was stored in aliquots under sterile conditions without preservatives at $-70^{\circ} \mathrm{C}$. When used after storage. the plasma samples were centrifuged before use. Plasma pools were prepared by mixing equal amounts of stored plasma from low responder cystic fibrosis (LRCF) patients or from normal individuals. Mixtures of normal and LRCF plasma were prepared by adding 5, 10 , and $15 \%$ of CF heat-inactivated plasma to autologous normal plasma to a total plasma concentration of $20 \%$. PA-absorbed plasma was prepared by mixing plasma thoroughly with $0.05 \mathrm{ml}$ of packed killed PA. After $30 \mathrm{~min}$ incubation at $37^{\circ} \mathrm{C}$, the mixture was spun $30 \mathrm{~min}$ at $1600 \times \mathrm{g}$. The supernatant plasma, shown to be devoid of agglutinating activity for PA, was used as "absorbed plasma."

\section{STATISTICAL METHODS}

Results are given as net cpm (mean cpm over background for triplicate cultures). For each series of antigen dilutions, the highest mean of the optimal response to both strains tested was used. For experiments in which specific variables of the culture conditions were tested, the percentage response compared to control response was calculated (experimental cpm: control cpm $\times 100)$. In addition, stimulation indices $(\mathrm{SI}=$ experimental $\mathrm{cpm}$ : background $\mathrm{cpm}$ ) were calculated when modifications of the culture system resulted in background $\left[{ }^{3} \mathrm{H}\right]$ thymidine incorporation changes. Statistical analyses were performed using the Student $t$ test for paired variables. $P$ 's are indicated only when $<0.05$.

\section{RESULTS}

\section{PLASMA SUBSTITUTION EXPERIMENTS}

The effect of substitution of homologous for autologous plasma on the lymphocyte responses to PA is shown in Table 1. Both C F and normal low responders increased their responses significantly when cultured in homologous normal plasma. There were only three normal individuals with low responses that could be tested. The effect of different plasmas on the same normal lymphocytes showed variable individual increases in responsiveness. Only in one of 13 instances did the response not increase over 2000 net $\mathrm{cpm}$. This is in contrast with the results obtained with $19 \mathrm{LRCF}$ lymphocytes. Although there was a significant increase in the responsiveness to $\mathrm{PA}$ in the group as a whole. 10 of the 19 patients did not increase their response to greater than 2000 net cpm. Only two of the nine patients increased their responses about 8000 net $\mathrm{cpm}$. The mean response for normal individuals in autologous plasma is $8510 \pm 1323$ net $\mathrm{cpm}$. A similar increase in response was not observed when individuals with low response to Staphylococcus in autologous plasma were cultured in homologous plasma.

The effect of CF plasma on the responses of normal and homologous CF lymphocytes is shown in Table 2. Plasma obtained from low responder CF patients did not inhibit the responses of normal lymphocytes to PA nor did it further decrease the responses to $\mathrm{CF}$ lymphocytes. Background values of normal

Table 1. Effect of homologous normal plasma on lymphocvte responses to Pseudomonas I influence of degree of responsiveness in autologous plasma

\begin{tabular}{|c|c|c|c|c|}
\hline \multirow[b]{2}{*}{ Source of lymphocytes } & \multirow[b]{2}{*}{ Source of plasma } & \multicolumn{3}{|c|}{ Degree of response in autologous plasma (net cpm) } \\
\hline & & $\operatorname{Low}(<2(K) 0 \mathrm{cpm})$ & $\begin{array}{c}\text { Medium }(20000-8000 \\
(\mathrm{pm})\end{array}$ & High $(>8000 \mathrm{cpm})$ \\
\hline \multirow[t]{3}{*}{ Normal donors } & Autologous & $(3 \cdot 13)^{\prime} 1.3() 4 \pm 40^{2}$ & $(9-12) 4.108 \pm 429$ & $(10-26) 14,195 \pm 1,082$ \\
\hline & Homologous & $4.513 \pm 890$ & $5.114 \pm 1.122$ & $14.808 \pm 1.529$ \\
\hline & Normal platsmial & $P<0.0(0) 25^{3}$ & & \\
\hline \multirow[t]{5}{*}{ CF Patients } & Autologous & $(19) 703 \pm 133$ & (27) $4,111 \pm 239$ & (16) $10.824 \pm 6.57$ \\
\hline & Plasma & & & \\
\hline & Homologous & $3,178 \pm 811^{1}$ & $5.932 \pm 675$ & $10.132 \pm 1.129$ \\
\hline & Normal plasma & & & \\
\hline & & $P<0.005$ & $P<0.01$ & \\
\hline
\end{tabular}


Table 2. Effect of CF plasma on the responses of normal and CF lymphocytes to $P A$

\begin{tabular}{|c|c|c|c|c|}
\hline \multirow[b]{2}{*}{ Source of lymphocytes } & \multirow[b]{2}{*}{ Source of plasma } & \multicolumn{3}{|c|}{ Degree of responsiveness of CF plasma donors' (net cpm) } \\
\hline & & $\operatorname{Low}^{2}(<2000 \mathrm{cpm})$ & Medium $(2000-8000 \mathrm{cpm})$ & High $(>8000 \mathrm{cpm})$ \\
\hline \multirow[t]{4}{*}{ Normal donors } & Autologous & $(16)^{3} 7,807 \pm 1,164^{4}$ & (13) $8,241 \pm 1,660$ & (9) $11,031 \pm 2,330$ \\
\hline & Plasma & & & \\
\hline & Homologous & & & \\
\hline & CF plasma & $7,146 \pm 1,317$ & $8,078 \pm 1,493$ & $12,221 \pm 2,848$ \\
\hline \multirow[t]{4}{*}{ CF patients } & Autologous & (6) $3,400 \pm 1,129$ & (7) $4,921 \pm 1,846$ & (5) $2,967 \pm 802$ \\
\hline & Plasma & & & \\
\hline & Homologous & & & \\
\hline & CF plasma & $3,442 \pm 936$ & $4,939 \pm 1,162$ & $5.720 \pm 3,489$ \\
\hline
\end{tabular}

lymphocytes incubated with CF plasma did not show a significant variation in comparison to background values in autologous plasma. Table 3 summarizes the results obtained with plasma substitution experiments on LRCF patients and normal controls. Although CF plasma from the three groups of patients increase the responses of normal lymphocytes to Staphylococcus, the differences are not significant (Table 4). The changes of responses to Staphylococcus and to PA induced by individual plasmas were not correlated.

The use of pooled normal or low responder CF plasma did not give different results from those obtained with large numbers of individual plasma-lymphocyte combinations. Both normal and CF lymphocytes with low responses in autologous plasma increased their responses to PA when homologous plasma pools were used.

\section{ABSORPTION EXPERIMENTS}

These experiments were performed to determine if inhibitors in CF plasma could be removed by preincubation with PA. Three LRCF plasma samples and three normal control plasma samples were absorbed with antibiotic killed PA and then used in experiments with autologous lymphocytes. When absorbed, normal plasma was used, previously responding lymphocytes became unresponsive to PA. Absorption of LRCF plasma did not increase the autologous CF lymphocyte responses. The SI for both normal and LRCF individuals in PA absorbed plasma were $\leq 2$, indicating that no lymphocyte activation by PA occurs under these conditions. By contrast, in both normal individuals and patients, the SI of the responses to Staphylococcus in PA absorbed plasma ranged from 12 to 63 .

The high concentration of specific PA antibodies in CF plasma is likely to change the antigenicity of PA. To test this possibility, the PA used to absorb LRCF was resuspended to original v/v concentrations and used as antigen. The effect of the preincubation of killed PA in LRCF plasma on its antigenicity is shown in Table 5. PA incubated in LRCF plasma increased the responses of normal lymphocytes. Lymphocytes from two of three LRCF patients showed a moderate increase in their responses. Similar increases were observed with PA preincubated with plasma from high responder CF patients or normal individuals.

Normal and LRCF plasma mixtures were used to test whether an inhibitory factor in CF plasma could lose activity when diluted in normal plasma. An increase in activity in plasma mixtures indicates complementary function of factors present in both types of plasma. Experiments were performed by incubating lymphocytes from five high-responding normal individuals in $20 \%$ autologous plasma, LRCF plasma, or plasma mixtures. In all instances, an increased responsiveness to PA was observed with plasma mixtures compared to autologous or LRCF plasma alone. The
Table 3. Summary of plasma effects on lymphocyte proliferative responses to $P A$ in $L R C F$ patients and normals

\begin{tabular}{lll}
\hline & LRCF plasma & \multicolumn{1}{c}{ Normal plasma } \\
\hline LRCF lymphocyte & Low or absent & $\begin{array}{c}\text { Moderate increase in } \\
\text { only 50\% of cases }\end{array}$ \\
Normal lymphocyte & Normal & Normal \\
\hline
\end{tabular}

increases ranged from 8 to $23 \%$ over the responses in autologous plasma and from 29 to $211 \%$ over the responses in $20 \%$ homologous LRCF plasma alone. The peak increase with plasma mixtures was usually found with either 5 or $10 \%$ LRCF plasma.

To determine if CF plasma factors interfered with early events in the triggering of lymphocyte proliferation by PA antigens, lymphocyte cultures were started in the absence of plasma. Plasma was then added $60 \mathrm{~min}$ after antigen addition to the cell suspension. Some control cultures had plasma included before addition of antigen. Others were kept in plasma-free conditions throughout the experiment. Cultures to which no plasma was added over the 5 days incubation period showed proliferative responses to mitogens (phytohemagglutinin, concanavalin $\mathrm{A}$, and pokeweed mitogen) and Staphylococcus. No response to PA was found under plasma-free culture conditions, in normal individuals, or in CF patients. The delayed addition to plasma after incubation of lymphocytes and bacterial antigens for $60 \mathrm{~min}$ allowed responses of normal individuals to PA antigens, but did not increase the responses of three LRCF patients.

Preincubation of lymphocytes was performed to allow shedding or pinocytosis of blocking complexes on the lymphocyte surface. Two types of experiments were performed. In short-term preincubation studies, lymphocytes were kept in plasma-free culture medium at $37^{\circ} \mathrm{C}$ for 30 min before cultures were set up in normal heat-inactivated plasma. This preincubation was shown to reduce the amount of immunoglobulin-bearing lymphocytes by 20 to $80 \%$. None of the four LRCF patients increased their responses to PA under these conditions. In a second group of experiments, the preincubation was performed in plasma-poor medium for prolonged periods of time. Lymphocytes were kept at $37^{\circ} \mathrm{C}$ in $1-\mathrm{ml}$ aliquots in RPMI medium and $2 \%$ heat-inactivated plasma for 24 and $48 \mathrm{hr}$ and for 5 days. The cells were resuspended daily in fresh medium. At the completion of the preincubation period, lymphocytes were resuspended in fresh medium. Viable cells were counted, readjusted to $1 \times 10^{6}$ cells $/ \mathrm{ml}$, and cultured for additional 5 days in the presence of antigens. Cells from normal individuals and from responding $\mathrm{CF}$ patients maintained their capacity to respond to Staphylococcus and PA antigens after incubation for up to 5 days, with daily changes of culture medium. None of the 
Table 4. Influence of CF plasma on the response of normal lymphocytes to Staphylococcus

\begin{tabular}{ccccc}
\hline \multirow{2}{*}{$\begin{array}{c}\text { Source of lympho- } \\
\text { cytes }\end{array}$} & Source of plasma & \multicolumn{3}{c}{ Degree of responsiveness to Pseudomonas of CF plasma donors } \\
\cline { 3 - 5 } & +Autologous & (12) $40,984 \pm 7,587^{\prime}$ & (13) $37,271 \pm 4,926$ & High $(>8000 \mathrm{cpm})$ \\
\hline Normal donors & +Homologous CF plasma & $65,729 \pm 6,225$ & $44,050 \pm 4,990$ & $(9) 52,254 \pm 8,487$ \\
\hline
\end{tabular}

${ }^{\prime}$ Mean \pm S.E.

Table 5. Effect of preincubation of $P A$ in low responder of plasma on lymphocyte responses to $P A$

\begin{tabular}{ccc}
\hline & $\begin{array}{c}\text { Untreated PA } \\
\text { (net cpm) }\end{array}$ & $\begin{array}{c}\text { PA preincubated } \\
\text { with LRCF } \\
\text { plasma (net cpm) }\end{array}$ \\
\hline Normal Lymphocytes & & \\
Control 1 & 4,763 & 9,336 \\
Control 2 & 20,416 & 24,592 \\
Control 3 & 8,881 & 12,158 \\
LRCF Lymphocytes & 359 & 374 \\
Patient 1 & 146 & 1,150 \\
Patient 2 & 163 & 2,326 \\
Patient 3 & \multicolumn{2}{c}{$P<0.01$} \\
\end{tabular}

eight LRCF patients studied increased their responses to PA after 1,2 , or 5 days preincubation. Control responses to staphylococci were present in all instances under these conditions. In two additional experiments, CF lymphocytes were preincubated for 24 hr in RPMI medium and 1\% human albumin only, with similar results.

\section{DISCUSSION}

In previous studies, we have shown that in vitro lymphocyte responses to PA are low or absent in CF patients with advanced disease. In some instances, these responses could be increased by substituting normal for CF plasma (24). Here we show that this increase of low responses, rather than being specific for $\mathrm{CF}$ lymphocytes, is also observed in low responder normal lymphocytes. Thus, plasma appears to exert a regulatory effect on lymphocyte responses to PA both in CF patients and normal individuals. Experiments performed under plasma-free conditions show that the response to PA is strictly plasma dependent. Unlike responses to PA, lymphocytes do respond to Staphylococcus, and to the mitogens phytohemagglutinin, concanavalin $A$, and pokeweed mitogen in plasma-free conditions. Further evidence for the plasma dependence of the lymphocyte response to PA was gained by absorbing plasma with killed PA. This absorption rendered plasma completely unsupportive of lymphocyte reactivity to PA. The specificity of this effect was shown by the fact that responses to staphylococci occur in the presence of PA absorbed plasma. In accordance with the lack of response to PA in absorbed plasma, PA preincubated with unabsorbed plasma becomes a stronger antigen than the untreated PA control.

Several CF factors have been found in serum from CF patients. One group of these factors appears to be genetically determined and has been found in homozygous as well as heterozygous individuals. These factors are inactivated by heat and by heparin $(4,6)$ and are not present in the heat-inactivated heparinized plasma used in our assay. A heat-stable factor has been shown to inhibit PA uptake by alveolar macrophages (26). A partially heatstable CF plasma factor was shown to inhibit the increase of total protein and $\beta$-glucuronidase in CF lymphocytes stimulated with phytohemagglutinin (13). We failed to see a similar effect of CF plasma on PHA-induced $\left[{ }^{3} \mathrm{H}\right]$ thymidine incorporation (24). Here we show that heat-inactivated CF plasma does not inhibit $\left[{ }^{3} \mathrm{H}\right]$ thymidine incorporation of either normal or homologous $\mathrm{CF}$ lymphocytes in response to bacterial stimulation. Although reduc- tions of responsiveness are seen in some combinations of $\mathrm{CF}$ plasma with normal or CF lymphocytes, increased responses are observed as well. Under certain circumstances, LRCF plasma consistently increases responses to lymphocytes to PA. When bacteria are preincubated with this plasma, they elicit higher responses in normals and also increase responses to PA of LRCF lymphocytes. The increased responses in the presence of $\mathrm{CF}$ and normal plasma mixtures indicates that, rather than inhibitors, $C F$ plasma contributes factors which collaborate with normal plasma factors in eliciting a lymphocyte response to PA. These results are in keeping with our earlier observation that the presence of PAagglutinating antibodies did not correlate with lymphocyte unresponsiveness (23). Hoiby et al. (10) had shown that CF plasma with multiple precipitins to PA antigens did not interfere with the responses of normal lymphocytes to heat-killed PA, soluble PA antigens, and heat killed Escherichia coli (10). In this paper, we present additional evidence against an inhibiting effect of antibodies by showing that their absorption out of CF plasma does not restore lymphocyte responsiveness to PA.

Several findings in this paper support the concept that the defective responses to PA in vitro are due to cellular factors rather than heat-stable plasma inhibitors present in CF patients. Only $50 \%$ of low responder $\mathrm{CF}$ patients increase their lymphocyte responses to PA in normal plasma. Moreover, the observed increases do not reach to the level of responses seen in normal individuals. To rule out the inhibition of lymphocytes by $\mathrm{CF}$ factors or immune complexes adhered to their surface, cells were incubated and washed for up to 5 days before stimulation with PA $(7,17,30,31)$. No recovery or increase of responsiveness was seen under these conditions. A similar finding had been reported earlier by Hoiby et al. (10). Repeated washing of CF lymphocytes failed to increase their responses to microbial antigens.

The presented evidence does not rule out the possibility that immune complexes play a role in creating a state of unresponsiveness of CF lymphocytes in vivo. Immune complexes are likely to occur in CF patients who have high specific antibody titers for PA $(11,20)$ together with heavy airway infection with these bacteria $(5,14)$. Several reports confirm their presence in CF serum $(18,19,25)$. Experimentally induced tolerance to orally administered antigens is based on the presence of such complexes (1). Similar mechanisms could operate for antigens presented to the bronchopulmonary lymphoreticular system. Moreover, immune complexes have been shown to be required for the expression of suppressor function by suppressor T-cells (16). Our work has been carried out with heat-inactivated plasma as conventionally used for lymphocyte culture assays. Heat inactivation is known to alter immune complexes and destroy them if they are heat labile $(21,34)$. Therefore, it is conceivable that immune complexes in vivo alter monocyte and lymphocyte subpopulations in such a way that the phenomona become irreversible to incubation, washing, or plasma substitutions in vitro. The in vivo reversibility of the lymphocyte unresponsiveness observed in some CF patients after hospitalization and intensive antibiotic therapy is compatible with this hypothesis.

Although further experiments using fresh CF serum or purified heat labile CF-factors are required for a definitive answer, it is unlikely that they cause the lymphocyte unresponsiveness to PA. Their presence is genetically determined and independent of progression of disease or severity of PA infection $(3,29)$. In contrast, lymphocyte unresponsiveness is associated with advanced disease and PA infection (22-24). Experiments in which 
CF lymphocytes were incubated in a low concentration of heatinactivated plasma or in complete absence of CF plasma provide further evidence against in vivo blocking of lymphocyte receptors by heat-labile CF factors.

A strong macrophage inhibiting factor has been described in the serum of CF patients (2I). Although we have not observed a similar plasma effect on the in vitro lymphocyte responses to PA, both mechanisms could play a role in the pathogenesis of lymphocyte unresponsiveness to PA. Benacerraf and Germain (2) have shown that in mice, the response to the synthetic copolymer $L$-glutamic acid ${ }^{60}-L$-alanine $e^{301}-L-$ tyrosine ${ }^{111}$ depends on the presentation of the antigen (2). The presenting cells are most likely macrophages. In L-glutamic acid ${ }^{(j \mid 1}-L-$ alanine ${ }^{:(x)}-L-t y r o s i n e^{101}$ responder strains of animals, T-helper cells are generated by this antigen. In nonresponder animals or in responding animals in which there is interference with the presentation of the antigen by the macrophage, $\mathrm{T}$-suppressor cells develop instead of $\mathrm{T}$-helper cells. It is, therefore, conceivable that in CF patients, abnormal macrophage function results in lymphocyte subpopulation changes in such ways that proliferative responses to PA are abrogated. This is a testable hypothesis which is currently being investigated in our laboratory.

\section{REFERENCES AND NOTES}

I. Andre. C.. Heremans, J. F.. Vaerman, J. P.. and Cambiaso, C. L.: A mechanism for the induction of immunological tolerance by antigen feeding: antigenantibody complexes. J. Exp. Med.. 142: 1509 (1975).

2. Benacerraf. B., and Germain, R. N.: Specific suppressor responses to antigens under I region control. Fed. Proc., 38: 2053 (1979).

3. Creglendy Nagy. E.. Khan. S., and Sturgess. J. M.: Serum factor in cystic fibrosis: Correlation with clinical parameters. Pediatr. Res.. 1.3: 729 (1979).

4. Di Sant Agnese. P. A., and Davis. P. B.: Research in cystic fibrosis. N. Engl. J. Med.. 295: 481 (1976).

5. Doershuk. C. F., and Matthews. L. W.: Cystic Fibrosis and obstructive pulmonary disease. In: M. Green R. J. Haggerty: Ambulatory Pediatrics. pp. 707-726 (W. B. Saunders (o., Phildelphia, PA. 1968).

6. Doggett. R. G. and Harrison. G. M.: Cystic fibrosis: in vitro reversal of the ciliostatic character of serum and salivary secretions by heparin. Nat. New Biol., 243: 251 (1973).

7. Fitch. F. W.: Selective suppression of immune responses. Regulation of antibody formation and cell immunity by antibody. Prog. Allergy. 19: 195 (1975).

8. Green. G. M.: In defense of the lung (The J. Burns Amberson Lecture). Am. Rev. Respir. Dis., 102: 691 (1970).

9. Green, G. M., and Kass, E. H.: The role of the alveolar macrophage in the clearance of bacteria from the lung. J. Exp. Med.. 1/4: 167 (1964).

10. Hoiby. N.. Andersen, V.. and Bendixen. G.: Pseudomonas aeruginosa infection in cystic librosis. Humoral and cellular immune responses against Pseudomonas deruginosa. Acta Pathol Microbiol. Scand.. Sect. C Immunol., 83: 459 (1975)

11. Hoiby. N., and Wiik. A.: Antibacterial precipitins and autoantibodies in serum of patients with cystic fibrosis. Scand. J. Respir. Dis., 56: 38 (1975).

12. Kaltreider. H. B.: Expression of immune mechanisms in the lung. Am. Rev. Respir. Dis.. 113: 347 (1976)

13. Lieberman. J, and Kaneshiro, W.: Abnormal response of cultured lymphocytes to phytohemagglutinin and autologous serum in cystic fibrosis. Am. Rev. Respir. Dis., 116: 1047 (1977).

14. Mearns, M. B., Hunt. G. H., and Rushworth, R.: Bacteria flora of respiratory tract in patients with cystic fibrosis 1950-1971. Arch. Dis. Child., 47: 902 (1972).

15. Moore. V. L.. and Myrvik. Q. N.: The role of normal alveolar macrophages in cell mediated immunity. RES J. Reticuloendothel. Soc., 21: 131 (1977).

16. Moretta. L.. Webb. S., Grossi, C.. Lydyard. P. M. and Cooper. M.: Functional analysis of two human $T$-cell subpopulations: help and suppression of B-cel responses by $\mathrm{T}$-cells bearing receptors for $\lg M$ or $\operatorname{lgG}$. J. Exp. Med.. 146: 184 (1977).
17. Oberbarnscheidt, J., and Kolsch, E.: Direct blockade of antigen-reactive B lymphocyles by immune complexes. An off signal for precursors of IgM producing cells provided by the linkage of antigen and Fc receptors. Immunology, 35: 151 (1978)

18. Schoitz. P. O., Hoiby. N.. Juhl. F.. Permin. H.. Nielsen. H., and Svehag. S-E: Immune complexes in cystic fibrosis. Acta Pathol. Microbiol. Scand. Sect. C Immunol. 85: 57 (1977).

19. Schiotz, P. O.. Nielsen, H., Hoiby, N., Glickman, G.. and Svehag. S-E: Immune complexes in the sputum of patients with cystic fibrosis suffering from chronic Pseudomonas aeruginosa lung infection. Acta Pathol. Microbiol. Scand. Sect. C Immunol., 86: 37 (1978).

20. Schwartz, R. H.: Serum immunoglobulin levels in cystic fibrosis. Am. J. Dis. Child.. III: 408 (1966).

21. Soltis, R. D., Hasz, D., Morris, M. J., and Wilson, I. D.: The effect of heat inactivation of serum on aggregation of immunoglobulins. Immunology. 36 37 (1979).

22. Sorensen, R. U., Stern. R. C., Chase. P., and Polmar, S. H.: Defective cellular immunity to gram-negative bacteria in cystic fibrosis patients. Infect. Immun.. 23: 398 (1979).

23. Sorensen. R. U.. Stern. R. C.. and Polmar, S. H.: Cellular immunity to bacteria Impairment of in vitro lymphocyte responses to Pseudomonas aeruginosa in cystic fibrosis patients. Infect. Immun., 198: 735 (1977).

24. Sorensen, R. U., Stern, R. C.. and Polmar. S. H.: Lymphocyte responsiveness to Pseudomonas aeruginosa in cystic fibrosis: relationship to status of pulmonary disease in sibling pairs. J. Pediatr., 93: 201 (1978).

25. Soter, N. A., Mihm. M. C.. and Colten. H. R.: Cutaneous necrotizing vasculitis in patients with cystic fibrosis. J. Pediatr., 95: 197 (1979).

26. Thomassen. M. J., Boxerbaum. B.. Demko. C. A.. Kuchenbrod. P. J.. Dearborn. D. G.. and Wood. R. E.: Inhibitory effect of cystic fibrosis serum on pseudomonas phagocytosis by rabbit and human alveolar macrophages. Pediatr. Res.. 1.3: 1085 (1979).

27. Thorsby, E., and Bratalie, A.: A rapid method for preparation of pure lymphocyte suspensions. In: P. I. Terasaki: Histocompatibility Testing. pp. 655-656 (Munksgaard. Copenhagen. 1970).

28. Warr, G. A., and Martin. R. R.: Responses of human pulmonary macrophages to migration inhibition factor. Am. Rev. Respir. Dis.. 108: 371 (1973).

29. Wilson. G. B. and Fudenberg. H. H.: Studies on cystic fibrosis using isoelectric focusing. IV. Distinction between ciliary dyskinesia activity in cystic fibrosis and asthmatic sera and association of cystic fibrosis protein with the activity in cystic fibrosis serum. Pediatr. Res., 11: 317 (1977).

30. Winchester, R. J., Fu. S. M., Hoffman, T.. and Junkel. H. G.: IgG on lymphocyte surfaces: technical problems and the significance of a third cell population. J. Immunol. 1/4: 1210 (1975).

31. Winchester, R. J., Winfield. J. B.. Siegal. F.. Wernet, P.. Bentwich, Z, and Kunkel. H. G.: Analysis of lymphocytes from patients with rheumatoid arthritis and systemic lupus erythematosis. Occurrence of interfering cold-reactive antilymphocyte antibodies. J. Clin Invest.. 54: 1082 (1974).

32. Wood. R. E., Wanner. A., Hirsch. J., and Farrell. P. M.: Tracheal mucociliary transport in patients with cystic fibrosis and its stimulation by terbutaline. Am. Rev. Respir. Dis.. 111: 733 (1975).

33. Yeates. D. B.. Sturgess. J. M. Crozier. D.. Levison. H... and Aspin. N.: Mucociliary transport in the trachea of patients with cystic fibrosis. Arch. Dis. Child.. 5/: $28(1976)$.

34. Zubler. R. H.. Lange. G., Lambert, P. H., and Miescher. P. A.: Detection of immune complexes in unheated sera by a modified ${ }^{121} \mathrm{I}-\mathrm{C}_{\mathrm{g}}$ binding test. Effect of heating on the binding of Clg by immune complexes and application of the test to systemic lupus erythematosis. J. Immunol., /16: 232 (1976).

35. Signed informed consent was obtained from patients and normal controls. The research protocol was approved by the Institutional Review Board for Human Investigation.

36. Requests for reprints should be addressed to: Ricardo U. Sorensen, M.D.. Departments of Pediatrics and Pathology. Case Western Reserve University School of Medicine. Rainbow Babies and Childrens Hospital. 2101 Adelbert Road. Cleveland. OH 44106 (USA).

37. This research was supported by National Institutes of Health grants HL-24244 and $A \mid 14682$ and grants from the Cystic Fibrosis Foundation and Health Foundation of Greater Cleveland.

38. Received for publication October 16, 1979

39. Accepted for publication April 18. 1980. 